\title{
Expectativa de logro, atribución y variables fisiológicas
}

\author{
Amparo Carpi Ballester, Consolación Gómez Iñiguez, \\ Cristina Guerrero Rodríguez, y Francisco Palmero Cantero \\ Universitat Jaume I de Castellón
}

\section{RESUMEN}

En este trabajo se presentan los resultados de la relación entre las variables cognitivas (expectativas de logro, nivel de preparación y atribución) con el rendimiento y con las variables fisiológicas (actividad cardiovascular) en una situación de evaluación académica. La muestra está integrada por 66 estudiantes universitarios, predominantemente mujeres, que cursan segundo año de la licenciatura de Psicología, con edades comprendidas entre 19 y 23 años (media $=20,1$ y $D T=1,38$ ). La obtención de información sobre las variables cognitivas se realizó antes y después de la ejecución del examen. Durante la realización del mismo, se registraron las variables fisiológicas en cada una de las fases: adaptación, tarea y recuperación. Se realizaron análisis descriptivos, de correlación, de regresión y ANOVA 2 (alta-baja: expectativas de resultado, nivel de preparación y atribución) x 3 (fases: adaptación, tarea y recuperación). En los resultados se observan diferencias significativas en la actividad fisiológica (presión sistólica [PS] y presión diastólica [PD]) entre los grupos con alta-baja expectativa de resultado y nivel de preparación.

\section{Palabras Clave}

Expectativa de nota, atribución causal, variables fisiológicas.

\section{AbstRact}

This paper presents the results of the interaction between cognitive variables (achievement and attribution) and physiological variables (cardiovascular activity) in a situation context of academic assessment. The participants were 66 university students, predominantly women, in the second year of the Psychology degree. They were between 19 and 23 years old (mean $=20.1$ and $S D=1.38$ ). The information on the cognitive variables was obtained before and after taking an exam. During the exam, the physiological variables were registered for each one of the phases: adaptation, task and recovery. We carried out descriptive, correlation and ANOVA 2 (highlow result expectation and perception of the preparedness for the exam) x 3 (adaptation, task, recovery) analyses. In the results are observed significant differences in the physiological activity (systolic pressure [SP]) and diastolic pressure [DP]) between the groups with high-low expectation of result and level of preparation.

KEY WORDS

Echievement, attribution and physiological variables. 


\section{Introducción}

En la decisión de realizar una conducta intervienen distintos factores que orientan la disposición de llevar a cabo la acción y explican el grado de implicación en la consecución de los objetivos y metas a lograr. Aunque son diversos los ámbitos en los que las personas actuamos, en este trabajo nos centramos en el marco académico de la enseñanza superior $y$, más concretamente, en los factores moduladores referidos a la ejecución del examen.

Entre los componentes de la actividad académica, además de la asistencia a las clases y el estudio, la realización de un examen es una de las tareas habituales y primordiales del proceso de aprendizaje. A través de los exámenes se evalúa, mediante distintos criterios, el aprendizaje realizado y/o la comprensión y diferenciación de los conceptos fundamentales que integran la materia en cuestión. Aunque la evaluación del conocimiento de una materia hace referencia a un momento, la decisión de realizar el examen, la implicación e interés en conseguir un determinado objetivo, forman parte de un proceso motivacional continuo y dinámico en el que la persona tiene que realizar un conjunto de acciones (como preparación, evaluación- valoración, atribuciones) en la que están implicados tanto factores cognitivos como afectivos y biológicos que, en última instancia, le aproximarán o alejarán de la situación objetivo (Palmero, 2005). Así, ante la perspectiva del examen, se realizan diversos procesos cognitivos dirigidos a valorar la situación de examen en cuanto a la dificultad que entraña la materia, el esfuerzo que se tiene que realizar -tiempo de preparación-, desarrollo de expectativas de re- sultado, así como valorar la capacidad que tiene la persona para lograr el resultado deseado, etc. Dichos procesos cognitivos contribuyen a organizar y desplegar un conjunto de acciones instrumentales dirigidas a reducir la probabilidad de fracaso, a aumentar la autosatisfacción $y$, en ciertos casos, además, a obtener el reconocimiento de las personas del entorno más inmediato (aprobación social).

Los procesos cognitivos llevados a cabo sobre la acción a realizar conforman un conjunto de factores motivacionales que juegan un importante papel en el desarrollo y/o mantenimiento de cualquier tipo de conducta. Uno de los modelos que explican la conducta motivada, desde la perspectiva cognitiva, es el de Atkinson (1964). Desde este modelo se explica la motivación de acuerdo con las expectativas de alcanzar una meta y en el valor que la misma posee para la persona. La interacción de ambos factores (expectativa-valor) determinará la conducta motivada ante el examen. Además, en la tendencia a alcanzar una meta, la esperanza de conseguir el éxito está contrabalanceada por el miedo a fracasar en la consecución del objetivo. Dicho miedo al fracaso puede llevar a que la persona se marque unas expectativas menos elevadas a modo de protección ante la vergüenza por el posible fracaso en la consecución del objetivo. Según Atkinson, las expectativas de éxito varían según las tareas y la situación en que éstas han de llevarse a cabo. Así, en determinadas tareas, las expectativas pueden ser más elevadas que en otras, de acuerdo con la dificultad encontrada en la tarea y/o al miedo ante el riesgo de fracaso y que se ponga en duda la capacidad de la persona. 
De este modo, la tendencia a la acción estará modulada no sólo por lo que se pretende conseguir (expectativas de éxito) sino también por la tendencia de evitar el fracaso. En este sentido, cuando una persona tiene que enfrentarse a una situación de evaluación, desarrollará unas expectativas de resultado y estimará los riesgos de fracaso en su consecución, y lo que éste puede suponer De este modo, se modula el grado de motivación, pues se ponen en marcha y se mantienen aquellas conductas necesarias que faciliten la consecución del objetivo deseado (éxito) y la reducción del fracaso. El examen académico es una de estas situaciones, ya que se evalúa el conocimiento adquirido en la materia en un período de tiempo y, en cierto modo, se evalúa también la capacidad del alumnado en la adquisición del conocimiento requerido.

Si bien la importancia que las expectativas ejercen en el inicio de diversidad de conductas ha sido corroborada en diversos trabajos (Bruinsma, 2004; De la Torre y Godoy, 2003; Marshall y Brown, 2004) ésta no es la única variable que influye en el desarrollo de la conducta motivada debido a que el proceso motivacional es complejo y dinámico. En este sentido, para lograr un objetivo, se deberá realizar un conjunto de acciones que faciliten la materialización de las expectativas ante el mismo. A su vez, la intensidad y persistencia en las acciones estarán influenciadas por las experiencias previas en situaciones similares. Así, tras el resultado alcanzado, la persona realizará los correspondientes análisis atribucionales que le permitan ajustar las futuras acciones para lograr el objetivo deseado (Palmero, 2005): la consecución del éxito señalará a esa conducta como potencialmente apropiada, mientras que la obtención de un fracaso señalará a esa conducta como potencialmente inapropiada. En general, las teorías basadas en la atribución combinan características personales y ambientales para explicar las causas de la conducta. Así, el modelo enunciado por Heider (1958) distingue entre disposiciones internas a la persona (habilidades, intenciones, etc.) y factores situacionales o ambientales (dificultad de la tarea, suerte, etc.). Si bien este modelo intenta explicar la conducta motivada en cualquier ámbito de acción, Weiner $(1973,1980)$ explicó el desarrollo de la motivación a partir de las atribuciones realizadas en las situaciones de logro. Según Weiner, en una situación de logro, además de la interpretación causal interna (factores personales como habilidad y esfuerzo) y externa (factores ambientales, como suerte y dificultad) también se debe tener en cuenta la estabilidad e inestabilidad de las dimensiones anteriormente citadas. La atribución de los éxitos y/o fracasos puede realizarse de forma simple a una de estas dimensiones o bien de forma compleja, combinando las distintas dimensiones entre sí. De este modo, puede realizarse una atribución interna-externa relativamente estable (habilidad y dificultad de la tarea), interna-externa relativamente inestable (esfuerzo y suerte en la ejecución).

Teniendo en cuenta la complejidad del proceso motivacional, la reflexión realizada tras la ejecución de una conducta, buscando razones o causas que ayuden a explicar los resultados, tanto si son positivos -consecución de la metacomo si son negativos -no consecución de la 
meta-, contribuye al desarrollo y/o modificación de las futuras conductas y expectativas de logro (Heider, 1958; Weiner, 1986). Así, si la atribución es interna las personas desarrollarán las acciones pertinentes que les conduzcan a lograr lo deseado, mientras que, si se realiza una atribución externa, será más fácil que disminuya el interés y se abandone un objetivo. Por otra parte, el peso que tenga cada una de las dimensiones formuladas por Weiner (personales o ambientales) repercutirá, a su vez, tanto en el desarrollo de distintos estados emocionales (satisfacción, optimismo, orgullo, tristeza, vergüenza, indefensión) como en el grado de implicación en las tareas que faciliten la consecución de las metas deseadas. De este modo, si el resultado alcanzado es positivo (éxito), y se atribuye a factores personales, se desencadenarán sentimientos optimistas en cuanto a la capacidad, y se facilitará el mantenimiento y desarrollo de acciones apropiadas dirigidas a mantener o mejorar el resultado obtenido (De la Torre y Godoy, 2003). Por el contrario, asumiendo también la existencia de una atribución basada en factores personales, si los resultados son negativos (fracaso), se tenderá a modificar el esfuerzo en las conductas instrumentales previas, incrementándolas en el caso de que se tenga un elevado interés en lograr dicho objetivo, o bien se modificará o abandonará el objetivo a lograr.

En síntesis, dependiendo de la motivación y de la atribución causal realizada, interna-externa, se fortalecerá la creencia de capacidad persistiendo en las correspondientes actividades realizadas o bien disminuirá la capacidad percibida, debilitando o abandonando la ejecución de las conductas dirigidas al logro del objetivo.
Por otra parte, como en cualquier situación que implica una evaluación, el examen académico se ve afectado por variables afectivas cuya manifestación se reflejará en cambios de la respuesta fisiológica (Blasco, FernándezCastro, Doval, Moix, Rovira y Sanz, 1999; De la Peza y García, 2005; Do y Schallert, 2004; Gutiérrez y García, 1999), pudiendo influir ésta en la modificación de las expectativas y/o en el rendimiento. Tal como se constata en distintos estudios (Culler y Holahan, 1980; Gutiérrez, 1996; Gutiérrez y García, 1999; Rapee, 1993), como consecuencia de los cambios percibidos en la respuesta fisiológica, podría ocurrir que se procesase más fácilmente la amenaza o peligro de la situación que el contenido del material de aprendizaje, así como que el sujeto se percibiese con menor capacidad para hacer frente a las demandas de la situación, produciéndose una posible disminución del rendimiento. Parece que el interés en la incorporación de variables fisiológicas en la investigación de tareas referidas al ámbito académico (por ejemplo, un examen oral, hablar en público) queda restringido a la relación que mantienen dichas variables fisiológicas con otras variables psicológicas como la ansiedad y la deseabilidad social. En este sentido, en situaciones de estrés moderado/ alto, parece existir una relación negativa entre ansiedad elevada y rendimiento, así como una relación positiva entre ansiedad elevada y funcionamiento psicofisiológico; sin embargo, los trabajos más recientes evidencian cierta heterogeneidad en los resultados obtenidos (Beidel, Turner y Dancu, 1985; Derakshan y Eysenck, 2001; Gómez, Carpi, Guerrero 
y Palmero, 2006; Ekman y Shean, 1997; Meijer, 2001; Rohrmann, Netter, Hennig y Hodapp, 2003; Shwarzwzer y Buchwald, 2003).

No obstante, también nos parece prudente sugerir que el aumento de la respuesta fisiológica no siempre influye negativamente sobre el rendimiento, ya que en muchas ocasiones es necesaria la activación, incluso una activación notable, para que se realice la conducta. Así, la implicación en la tarea, el deseo de evitar el fracaso, entre otros factores, movilizará un conjunto de recursos personales, entre ellos los fisiológicos, que preparará a la persona para realizar la acción de la manera más óptima posible. La mayor respuesta fisiológica está dirigida a la movilización de recursos que pueden contribuir a un afrontamiento de la situación de forma más óptima y conseguir, de este modo, los resultados deseados. La activación fisiológica puede ser considerada como un recurso necesario para un funcionamiento cognitivo apropiado, siempre y cuando esa activación fisiológica sea adaptativa y no vaya más allá de los márgenes de control del individuo. En este sentido, y teniendo en cuenta el proceso motivacional, la expectativa de resultado positivo y la confianza en la propia capacidad para el desempeño de la tarea activarán un conjunto de recursos (afectivos, cognitivos y fisiológicos) dirigidos a realizar las acciones necesarias, lograr el objetivo deseado y evitar el fracaso (Gutiérrez y García, 1999; Palmero, 2005).

Atendiendo a la diversidad de factores que pueden incidir en la motivación de los estudiantes para preparar un examen, en este trabajo analizamos la influencia del nivel de preparación percibido en el desarrollo de las expectativas de rendimiento y en el rendimiento logrado; en qué medida las expectativas de rendimiento predicen el rendimiento obtenido; grado de relación entre la atribución informada con las expectativas de resultado, y la modulación de la respuesta fisiológica según las variables de expectativas de resultado, grado de preparación percibido y atribución.

De este modo, las hipótesis establecidas han sido las siguientes:

Los participantes con mayor percepción de preparación en la materia de examen manifestarán una expectativa de resultado más elevada que aquellos con menor preparación percibida.

Los participantes que puntúan más alto en grado de preparación de la materia atribuirán sus resultados a factores internos.

Los participantes con mayor expectativa de resultado presentarán una activación cardiovascular moderadamente más elevada durante la ejecución de la tarea, respecto a los que manifiestan menor expectativa de resultado.

La activación cardiovascular de los participantes con mayor expectativa de resultado se reducirá más rápidamente tras la ejecución del examen que aquellos con menor expectativa de resultado.

\section{Método}

\section{Muestra}

La muestra estuvo integrada por 66 estudiantes universitarios, en su mayor parte mujeres (54 mujeres y 12 hombres), con edades comprendidas entre 19 y 23 años (media = $20,1$ y $D T=1,38)$. 


\section{Procedimiento}

Tras informar a los estudiantes de la inmediata realización del presente experimento, consistente en la realización de un examen parcial de la asignatura "Psicología de la Motivación y Emoción", se solicitó su participación voluntaria. El examen a realizar durante el experimento presentaba la peculiaridad de llevarse a cabo de forma individual en el Laboratorio de Emociones y Psicofisiología Cardiovascular, donde el participante permanecía conectado a los sensores para medir su funcionamiento cardiovascular mientras ejecutaba dicho examen. En caso de aprobar el examen, el alumno eliminaba dicha materia del examen final. A su llegada al laboratorio, se les presentaba una hoja en la que se explicaba el procedimiento a seguir, tras lo cual los alumnos firmaban su consentimiento para participar en la investigación. Inmediatamente después, el alumno pasaba a la cabina experimental, en la que se colocaban los sensores y se les informaba de la duración, ambientación y características de la prueba que iba a realizar. Tras resolver las posibles dudas se iniciaba la sesión de registro. Al finalizar las distintas fases del experimento (adaptación, examen y recuperación) el participante abandonaba la cabina experimental. A continuación, en una sala contigua, el alumno, a modo de comprobación, revisaba las respuestas dadas al examen en formato de papel, que previamente el experimentador había anotado durante la fase de examen. Dicha revisión no conllevaba la modificación de las respuestas. Por último, se les agradeció su colaboración, indicándoles la fecha y el lugar para consultar la nota obtenida.

\section{Sesiones de registro}

Las sesiones de registro se realizaron de 9:00 h a 14:00 h en el Laboratorio de Emociones y Psicofisiología Cardiovascular de la Universidad Jaume I de Castellón. Previamente a la entrada en la cabina para realizar el examen, a cada participante se le preguntaba por la expectativa de nota y por el nivel de preparación de la materia a examinar. La escala de respuesta, tipo Likert, era de 10 puntos ( $1 \mathrm{a}$ 10). A continuación, el sujeto pasaba a la cabina acomodándose al sillón y se le conectaban los sensores para poder llevar a cabo el registro fisiológico (frecuencia cardiaca, presiones sanguíneas sistólica y diastólica). La duración de cada una de las fases fue: de 10 minutos para la fase de adaptación, en la cual no se presentaba ningún estímulo y se registraba la actividad fisiológica basal; de 20 minutos de duración para la fase de tarea, en la que se presentaban los estímulos (preguntas del examen) con un ritmo de una pregunta por minuto, permaneciendo los ítems visibles en la pantalla durante 30 segundos. El participante podía responder dentro de los 60 segundos; transcurrido dicho período, aparecía la siguiente pregunta. Tras la finalización del examen, se iniciaba la fase de recuperación, que es una situación de reposo en la que no hay estimulación, con una duración de $10 \mathrm{mi}$ nutos. El objetivo de la última fase es medir cuánto tarda el organismo en recuperar su nivel basal en la actividad cardiovascular (tasa cardiaca y presiones sistólica y diastólica). Tras la finalización del examen, se preguntaba de nuevo la expectativa de nota, con la finalidad de comprobar la influencia que la ejecución 
de la tarea puede presentar en la misma, y también se solicitó información sobre la atribución realizada; es decir, si el logro esperado se atribuía a factores personales (grado de preparación) o a factores externos. Las respuestas a los ítems sobre la atribución se realizaron también en una escala tipo Likert de 10 puntos.

\section{Material e instrumentos}

Se emplearon 4 ítems para medir las variables subjetivas (expectativas de resultado -antes y después de la ejecución del examen-, nivel de preparación percibido, atribución del resultado esperado) cuya escala de respuesta, tipo Likert, de 10 puntos (ver Apéndice). Las puntuaciones cercanas a 10 indicaban alta percepción de preparación, de expectativa y atribución interna, mientras las cercanas a 1 reflejaban baja percepción de preparación, de expectativa y atribución interna. Por el contrario, las puntuaciones intermedias reflejan una menor o débil convicción de cada una de las variables indicadas.

La tarea consistió en la realización de un examen, conformado por 20 preguntas con cuatro alternativas de respuesta (a, b, c, d). La fórmula de corrección del examen fue la siguiente: $[P=A-(E / k-1)) / K \times 10]$ siendo $A$ el número de aciertos, $E$ el número de errores, $k$ el número de alternativas, $K$ el número de preguntas. El resultado obtenido se multiplicará por 10 (nota máxima de examen) para calcular $P$ (puntuación del examen).

Los estímulos (preguntas de examen) fueron presentados mediante el programa SuperLab v. 2.0.2.
Además, se realizaron registros psicofisiológicos de las variables relacionadas con la actividad cardiovascular: frecuencia cardiaca (TC) -medida en latidos por minuto (lpm)-, presión sistólica (PS) y presión diastólica (PD) medidas en milímetros de mercurio $(\mathrm{mmHg})$. Los registros se llevaron a cabo mediante un sistema integrado BIOPAC MP 150 y el módulo NIBP $100 \mathrm{~A}$, permitiendo el registro de las respuestas fisiológicas de forma continua y no invasiva o incruenta. Para almacenar y visualizar los datos obtenidos, el equipo de registro fisiológico estaba conectado, a través de un convertidor analógico-digital, a un sistema informático.

\section{Diseño y análisis de datos}

Los datos obtenidos fueron analizados con el paquete estadístico SPSS (v. 15), realizándose análisis descriptivos, de correlación, de regresión y análisis de varianza.

Las puntuaciones directas obtenidas en las variables cognitivas (expectativas de nota, percepción de nivel de preparación y atribución) fueron transformadas, en variables dicotomizadas, tomando como punto de corte el valor de la mediana, para formar los respectivos grupos (alto-bajo) en cada una de las variables citadas.

\section{Resultados}

\section{Resultados descriptivos de la muestra}

\section{Expectativa de nota}

En relación con los resultados sobre la expectativa de nota informada antes de la realización del examen, el 74,2\% esperaba aprobar, el $21,2 \%$ de la muestra manifestó tener una ex- 
pectativa de suspenso (menos de 4 puntos), y sólo el 4,5\% esperaba lograr una puntuación de notable.

La expectativa de nota informada posteriormente a la realización del examen se modificó de la siguiente manera: aprobado un 54,5\%, suspenso un $39,4 \%$, y notable un $6,1 \%$ de la muestra. En ninguna de las expectativas informadas se obtuvieron respuestas acerca de lograr un resultado de sobresaliente.

A partir de los resultados, se puede observar que la ejecución de la tarea modifica las expectativas informadas sobre alcanzar el objetivo deseado.

En los siguientes diagramas de sectores se puede observar la distribución de las expectativas de nota informadas antes y después de la realización del examen.

Correlaciones y análisis de regresión entre las variables cognitivas y rendimiento.

Los análisis de correlación realizados entre las distintas variables predictivas -ver Tabla 1-, muestran el grado en que se relacionan entre sí y con la nota obtenida (logro) siendo dichas correlaciones distintas según se informe la expectativa antes o después de realizar el examen.

Así, el nivel de preparación percibido correlaciona significativamente con la expectativa de nota informada antes de la ejecución ( $r=0,673$; $p \leq 0,01$ ), siendo menor la correlación obtenida con la expectativa informada después de la ejecución del examen ( $r=0,390 ; p \leq 0,01)$. El menor valor de relación se repite de nuevo con la nota obtenida $(r=0,251 ; p \leq 0,05)$.

La expectativa de nota informada previamente a la realización del examen obtiene una correlación significativa con la nota obtenida

\section{Expectativa de nota antes del examen}

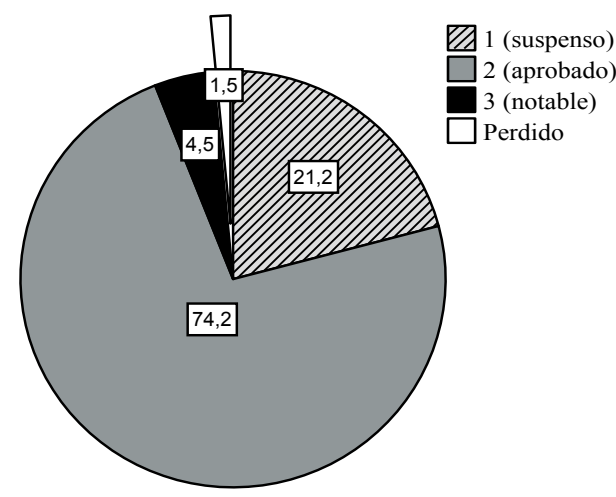

Expectativa de nota después del examen

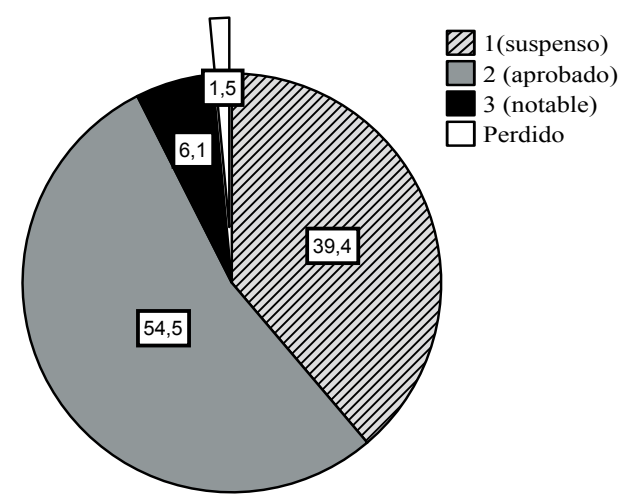

Figura 1. Distribución de la expectativa de nota.

\section{Nivel preparación percibido}

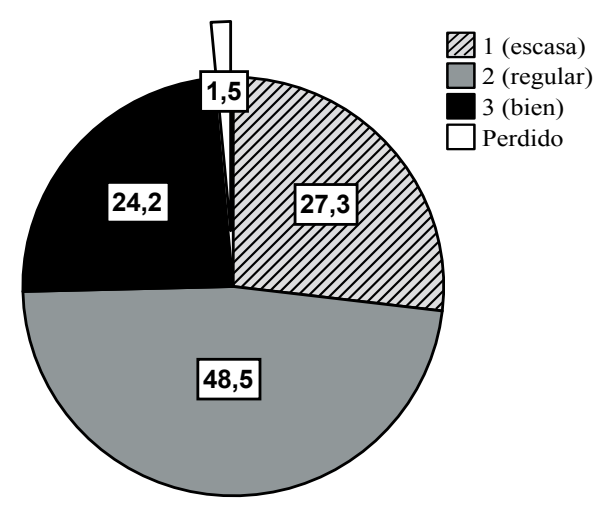

Figura 2. Distribución del nivel de preparación percibido. 
$(r=0,391 ; p \leq 0,01)$, siendo mayor la correlación obtenida con la expectativa de nota $(r=$ 0,$445 ; p \leq 0,01$ ) informada posteriormente a la ejecución del examen.

Las correlaciones obtenidas entre atribución interna con nivel de preparación $(r=0,199)$, con la expectativa de nota previa al examen $(r=0,092)$, con la expectativa de nota después del examen $(r=0,075)$ y con la nota obtenida $(r=0,24)$ carecen de significación estadística $(p>0,05)$.

A partir de la relación significativa encontrada entre las variables citadas en apartados anteriores (ver Tabla 1), observamos, mediante análisis de regresión y pruebas $\tau$, que el nivel de preparación percibido predice de forma significativa tanto la expectativa de nota informada antes $[\tau(65)=7,271 ; p \leq 0,01 ; \beta=0,673]$ y la informada después de la realización del examen $[\tau(65)=3,386 ; p \leq 0,01 ; \beta=0,390]$. Por otra parte, el rendimiento también es explicado por el nivel de preparación percibido $[\tau(65)$ $=2,076 ; p \leq 0,05, \beta=0,251]$. En relación con el rendimiento, observamos que la expectativa de nota informada antes de la ejecución del examen predice el rendimiento de modo satisfactorio $[\tau(65)=3,402 ; p \leq 0,01, \beta=0,391]$, al igual que la expectativa de nota informada después de la ejecución del examen $[\tau(65)=$ $3,974 ; p \leq 0,01, \beta=0,445]$. Esto es, las personas que perciben un mayor nivel de preparación desarrollan mayores expectativas de resultado y logran el objetivo deseado.

\section{Resultados de las variables fisiológicas}

Para analizar la modulación de las distintas variables cognitivas empleadas con las variables fisiológicas cardiovasculares se realizaron ANOVA 2 (grupos alto-bajo nivel de preparación, expectativas y atribución) x 3 (fases: adaptación-tarea-recuperación). Los registros fisiológicos (frecuencia cardiaca, presión sistólica y presión diastólica), tomados de forma continua a lo largo de las tres fases (adaptación, tarea y recuperación) han sido significativos en algunas de las relaciones citadas.

No se han obtenido diferencias estadísticamente significativas en la medida de la frecuencia cardiaca y cada uno de los grupos de las respectivas variables cognitivas. Dicha carencia de significación también se ha sido obtenida entre la variable de atribución y las medidas en presión sistólica (PS) y presión diastólica (PD).

Tabla 1

Correlaciones entre las variables cognitivas

(1)

(2)

(3)

(4)

(5)

(1) Nivel de preparación

1

(2) Expectativa de nota ANTES del examen

$0,673^{* *} \quad 1$

(3) Expectativa de nota DESPUÉS del examen

$0,390 *$

$0,664^{* *}$

$0,251^{*}$

$0,391^{* *}$

1

(4) Nota obtenida

0,199

0,092

0,075

0,024

1

* La correlación es significativa al nivel 0,01 (bilateral).

* La correlación es significativa al nivel 0,05 (bilateral). 
Sin embargo, los resultados entre las medidas en PS (E.T.m = 1,61 -adaptación-, 2,02 -tarea- y 1,64 -recuperación-) y en PD (E.T.m = 1,20 -adaptación-, 1,26 -tarea-y 1,23 -recuperación-), con la expectativa de nota, informadas antes y después de la ejecución del examen, y con el nivel de preparación percibido sí han sido estadísticamente significativos.

Los valores del factor expectativa de nota informada antes del examen en PS $[F(1,64)=8,252$;

Expectativa de nota previa al examen

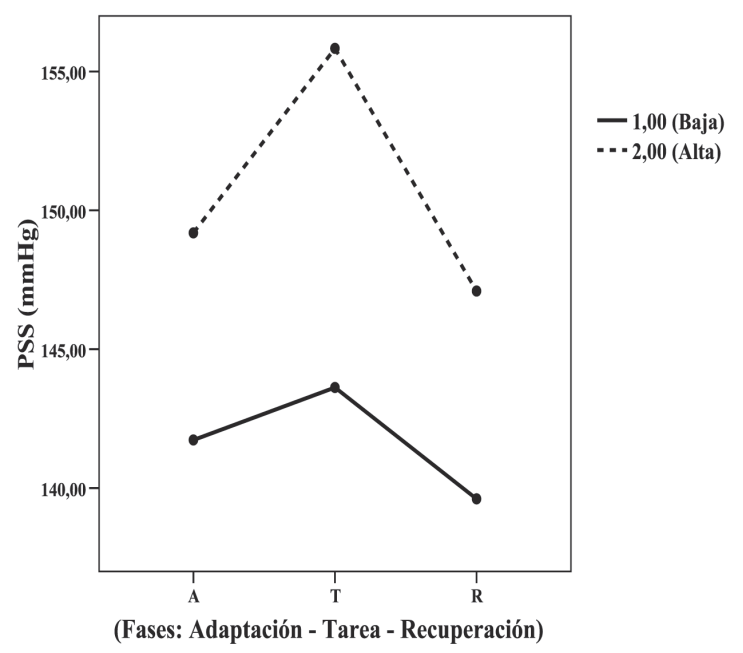

Figura 3. Expectativa previa - PS $(p \leq 0,01)$

Expectativa de nota previa al examen

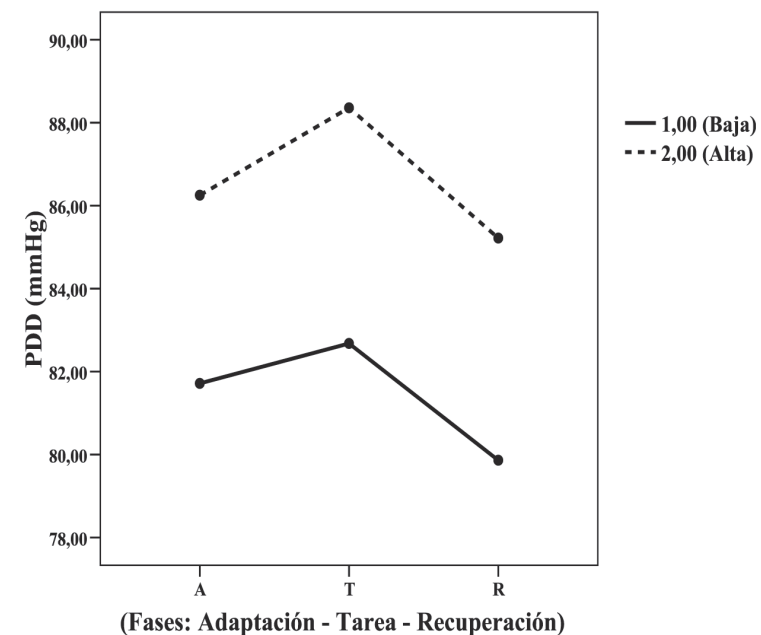

Fiigura 4. Expectativa previa - PD $(p \leq 0,01)$ $p \leq 0,01]$, y en PD $[F(1,64)=4,752 ; p \leq 0,05]$, indican la existencia de diferencias entre grupos para cada una de las fases. En las Figuras 3 y 4 se pueden observar las tendencias de los picos de activación alcanzados en ambos grupos (alta expectativa de nota versus baja expectativa de nota) respecto a cada una de las fases (adaptación, tarea y recuperación).

En el factor fases únicamente se obtuvo un valor marginalmente significativo en PS y la expectativa informada previamente a la ejecución $[F(1,64)=3 ; p \geq 0,05]$, apuntando dicha interacción que para los sujetos existe variación de activación en cada una de las fases. No obstante, las diferencias intrasujeto en PD $[F(1,64)=1,062 ; p \geq 0,05]$ en esta variable no ha sido estadísticamente significativa.

En cuanto a los valores obtenidos según grupo de alta-baja expectativa de nota informada después de la realización del examen y PS $[F(1,64)=$ $6,660 ; p \leq 0,01]$ y con PD $[F(1,64)=8,070 ; p \leq 0,01]$ también han sido estadísticamente significativos. En las siguientes figuras 5 y 6 se observan las tendencias de los picos de activación alcanzados

Expectativa de nota después del examen

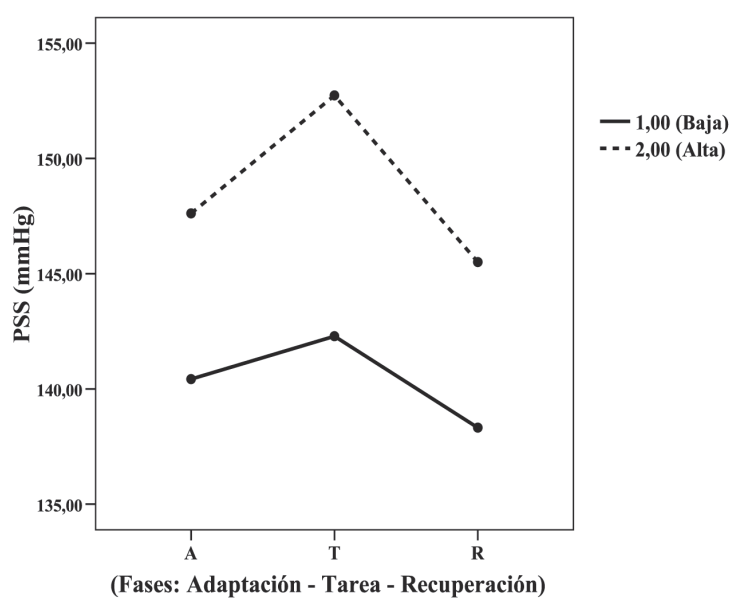

Figura 5. Expectativa posterior - PS $(p \leq 0,01)$ 
Expectativa de nota después del examen

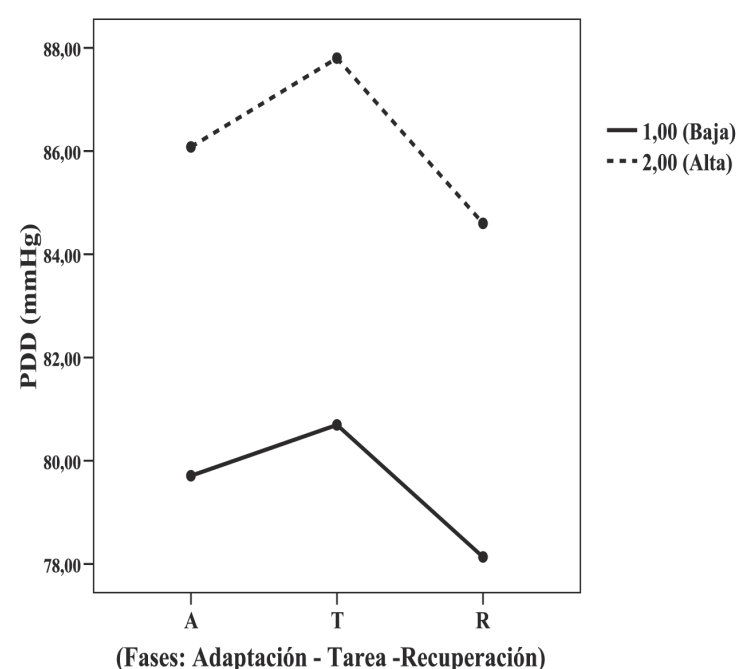

Figura 6. Expectativa posterior - PD $(p \leq 0,01)$

en ambos grupos (alta-baja expectativa $x$ fases -adaptación-tarea-recuperación).

Al igual que los resultados obtenidos en la expectativa de nota informada previamente al examen, las diferencias de activación según fases de la variable Expectativa informada después del examen en PS $[F(1,64)=1,368$; $\mathrm{p} \geq 0,05]$ y en $\operatorname{PD}[F(1,64)=0,922 ; p \geq 0,05]$, a nivel intragrupo, tampoco han sido significativas.

Así, las diferencias presentadas en las presiones sanguíneas dependen de la nota que cada grupo espera obtener: aprobado -alto- o suspenso -bajo-. Así, las tendencias encontradas en cuanto a la variación de la presión sanguínea, según las expectativas previas a la ejecución en cada una de las fases de la ejecución (adaptación, tarea y recuperación), denotan que la activación es mayor en los que esperan aprobar (alto) que en aquellos que esperan suspender (bajo), tal y como formulábamos en nuestra hipótesis de partida. Dicha activación aumenta, de forma más acusada, en los primeros durante la fase de tarea para disminuir, de manera no- toria, en la fase de recuperación. Sin embargo, en los que esperan suspender (bajo), aunque presentan un ligero aumento en la presión sanguínea durante la fase de tarea, este aumento no es tan elevado como en el grupo que espera aprobar (alto). La recuperación, aunque buena en los dos grupos, es más óptima en el grupo que espera aprobar.

En cuanto al nivel de preparación percibido e informado previamente a la ejecución del

Nivel de preparación percibido

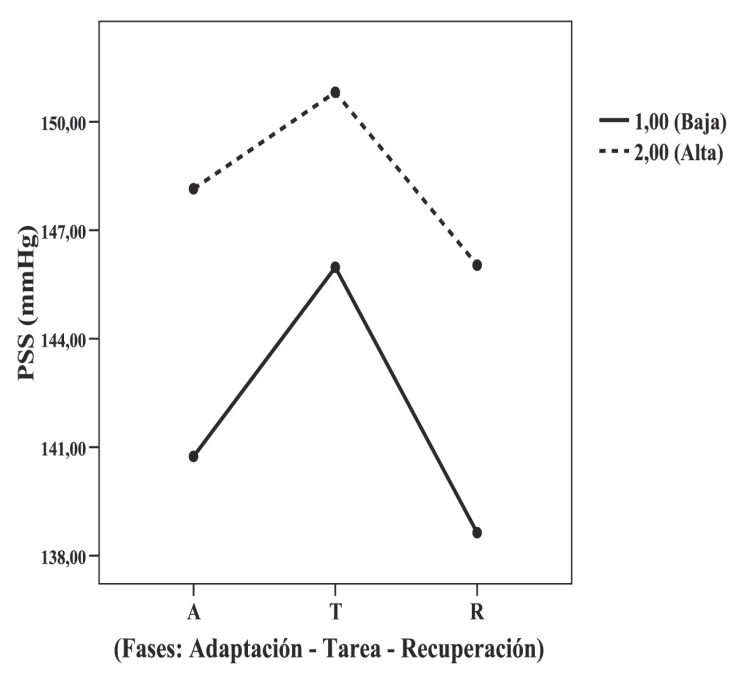

Figura 7. Nivel preparación - PS $(p \leq 0,05)$

Nivel de preparación percibido

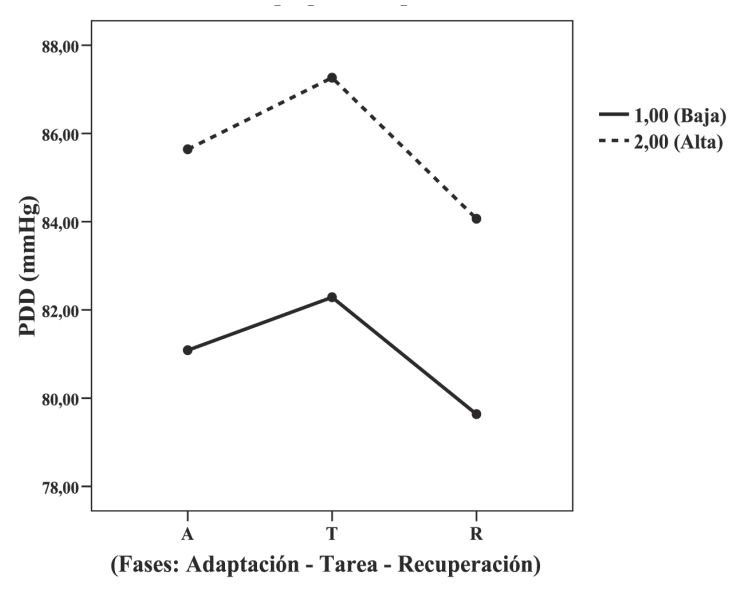

Figura 8. Nivel preparación - PD $(p \leq 0,05)$ 
examen, los resultados del ANOVA 2 (nivel de preparación: alto, bajo) x 3 (fases: adaptación, tarea, recuperación) los valores obtenidos en PS son $[F(1,64)=4,181 ; p \leq 0,05]$ y en $\operatorname{PD}[F(1,64)$ $=3,868 ; p \leq 0,05]$. Aunque en PD los resultados son marginalmente significativos consideramos óptimos los resultados obtenidos.

En las figuras 7 y 8 se muestran las tendencias de los picos de activación alcanzados en ambos grupos.

Como se observará, en las figuras, el grupo que manifiesta estar mejor preparado presenta un nivel de activación fisiológica mayor que los sujetos participantes que manifiestan menor nivel de preparación en cada una de las fases: adaptación, tarea y recuperación. En la fase de recuperación, el descenso de la activación que manifiestan los sujetos participantes que se perciben con menor preparación parece ser mejor que en los sujetos cuya percepción de preparación es más elevada. Es decir, les cuesta más recuperarse.

$\mathrm{Al}$ igual que en las variables anteriores (expectativa previa y posterior al examen), las diferencias de activación por fases a nivel intrasujeto no han sido estadísticamente significativas ni en PS $[F(1,64)=0,869 ; p \geq 0,05]$ ni en $\operatorname{PD}[F(1,64)=0,471 ; p \geq 0,05]$.

Tras los resultados obtenidos, en el siguiente apartado comentamos los aspectos más relevantes de acuerdo a las hipótesis planteadas en el estudio.

\section{Discusión}

En este trabajo se han analizado la influencia de la variable cognitiva nivel de preparación percibido en el desarrollo de las expectativas de rendimiento, y en qué medida el rendimiento logrado está modulado por las expectativas de resultado informado. Asimismo, se analizan los cambios en la respuesta fisiológica cardiovascular (frecuencia cardiaca, presión sanguínea sistólica y presión sanguínea diastólica) según las variables cognitivas empleadas: nivel de preparación percibido, expectativas de resultado y atribución informada, relacionada con lo que espera lograr en el examen.

A partir de los resultados se observa una relación predictiva significativa entre las variables cognitivas entre sí y con el rendimiento, y la modulación de la respuesta fisiológica cardiovascular (FC, PS y PD) con dichas variables cognitivas, permitiendo, de este modo, la confirmación parcial de alguna de las hipótesis planteadas. La variación de la respuesta cardiovascular (PS, PD) indica que ante la tarea aumentan la activación fisiológica para hacer frente a las demandas específicas, disminuyendo éstas tras la ejecución de la misma. La mayor respuesta fisiológica ante la tarea y la consecuente estabilización tras su ejecución apunta a que el perfil fisiológico manifestado es de carácter adaptativo.

Teniendo en cuenta que todas las hipótesis no se han cumplido como esperábamos, comentaremos los resultados obtenidos que nos permitan confirmar o rechazar cada una de las mismas.

Así, en relación con la primera de las hipótesis en la que se esperaba que a mayor nivel de preparación percibido se desarrollan mayores expectativas de rendimiento, los análisis efectuados informan que dicha variable predice satisfactoriamente ambas expectativas de re- 
sultado (informadas antes y después de la ejecución del examen), y que las diferencias en la expectativa de resultado queda explicada por el nivel de preparación percibido. A partir de los diferentes coeficientes de correlación obtenidos, podemos interpretar que las expectativas, informadas antes y después del examen, parecen indicar que la valoración realizada se ve afectada por el conocimiento de la tarea en cuestión. Es decir, previamente a conocer la tarea, la persona confía en la preparación realizada, y desarrolla, con mayor confianza, una expectativa de logro que, tras la ejecución del examen, y conociendo la dificultad que éste haya presentado, la preparación se percibe como menos óptima para conseguir el objetivo deseado. La misma interpretación queda reforzada a partir de los resultados obtenidos en las expectativas de resultado informados antes y después de la ejecución del examen. Basándonos en la teoría de Atkinson (1964), dichas expectativas se modifican de acuerdo con lo que realmente se está valorando; antes del examen, la referencia que se posee es el trabajo realizado, $y$, una vez conocida la dificultad concreta del examen, la persona tiene más elementos a valorar, concretamente la tarea objetivo que, al compararla con la preparación realizada, debe ajustar las expectativas. No obstante, podemos interpretar que el nivel de preparación percibido e informado previamente a la ejecución del examen permite desarrollar cierta confianza en la capacidad de lograr el objetivo deseado. Estos resultados apuntan en la misma dirección que en los obtenidos en estudios previos entre autoeficacia y percepción de logro en el ámbito académico
(Shell, Murphy y Brunning, 1989; Zimmerman, 1999), aunque dicha relación no ha sido confirmada en el conjunto en otros trabajos (Gore, 2006; Richard, Diefendorff y Martín, 2006), atribuyéndose la divergencia de resultados al modo de medir las creencias en la capacidad percibida. Con estos resultados, la primera de las hipótesis planteadas en el presente trabajo queda satisfactoriamente confirmada.

Sin embargo, los resultados no permiten apoyar la segunda de las hipótesis planteadas. Como se recordará, en la misma se esperaba que los participantes con mayor nivel de preparación manifestarían, posteriormente a la ejecución del examen, una atribución interna de los resultados esperados. Tanto los análisis de correlación como los de regresión no han sido estadísticamente significativos, por lo que no podemos confirmar la influencia predictiva del nivel de preparación percibido en la atribución realizada tras el examen. Estos resultados pueden ser debidos a la medida empleada de la atribución con un único ítem, que recogía, de modo general, si el rendimiento esperado se atribuía a la preparación o esfuerzo realizado. La existencia de otras características de la situación, como la dificultad encontrada en la tarea, la necesidad de protección ante el juicio del entorno por los resultados de fracaso esperado, entre otras, puede haber contribuido a este efecto. No obstante, hay que tener en cuenta que el alumnado tenía la posibilidad de recuperar posteriormente la materia examinada, por lo que el hecho de presentarse al examen no les suponía mayor riesgo, ya que les podía permitir un conocimiento acerca de la dificultad de la materia y prepararse poste- 
riormente de forma más adecuada. Así, muchos de ellos se presentaban al examen informando que esperaban suspender, por lo que estas personas pueden haber considerado que aprobar era cuestión de suerte y suspender no les suponía ningún riesgo de fracaso. Resultados similares se obtienen en otros estudios (González, Corral y Montaño, 2004; Manassero y Vázquez, 1995).

Además de los análisis de correlación y regresión realizados entre las variables cognitivas, en este trabajo también se ha analizado la influencia de estas variables en la modulación de la respuesta fisiológica cardiovascular (frecuencia cardiaca, presión sistólica y presión diastólica). Así, en la tercera de las hipótesis planteadas, se postula que los sujetos que presentan una mayor expectativa de resultado mostrarán mayor activación fisiológica en la fase de tarea que aquellos con menor expectativa. Dicha hipótesis se confirma parcialmente, ya que en frecuencia cardiaca las diferencias entre grupos no han sido significativas. Por el contrario, las diferencias en PS y PD entre los grupos con alta-baja expectativa de resultado sí han sido estadísticamente significativas, $y$, tal como expusimos en las Figuras 3 y 4, en la fase de tarea los sujetos que manifiestan lograr un buen resultado presentan una mayor activación que aquellos que presentan una baja expectativa de resultado. Así, se observa que la movilización de recursos fisiológicos es mayor en el grupo alto en expectativa de logro, y que dicha movilización es necesaria para afrontar la tarea lo más óptimamente posible.

Si la mayor movilización de recursos fisiológicos facilita abordar de forma óptima la tarea, tras la realización de la misma, cabe esperar que haya un restablecimiento de dicha activación, recuperándose los niveles basales previos de funcionamiento fisiológico, tal como indicamos en la cuarta de las hipótesis. Los resultados nos permiten, asimismo, confirmar la quinta de las hipótesis. No obstante, al igual que en los resultados fisiológicos anteriores, la recuperación esperada únicamente ha sido estadísticamente significativa en PS y PD. La recuperación fisiológica del grupo con mayor expectativa de resultado es mayor $y$ más rápida a la hora de volver a los niveles de funcionamiento anteriores a la tarea. Son resultados que coinciden con los obtenidos en una investigación previa (Guerrero y Palmero, 2006), pudiendo constatar que los individuos que perciben control sobre la tarea en una situación de estrés muestran valores psicofisiológicos superiores durante la fase de tarea y recobran antes sus niveles basales durante la fase de recuperación.

Aunque en estas hipótesis se ha hecho hincapié en las fases de tarea y recuperación, es necesario indicar que en la fase de adaptación la tendencia de respuesta fisiológica también es mayor en el grupo con mayor expectativa de logro. Por tanto, en esta fase, los sujetos empiezan a movilizar ya los recursos fisiológicos que les ponen en alerta para realizar de forma más apropiada la tarea requerida.

Los resultados significativos obtenidos en la última de las hipótesis nos permiten una confirmación parcial de la misma, ya que las diferencias de activación esperada sólo han sido significativas en PS y PD y no así en frecuencia cardiaca. Igualmente, los sujetos que manifies- 
tan un mayor nivel de preparación abordan la situación con mayores recursos fisiológicos que los que manifiestan nula o escasa preparación, recuperando su funcionamiento fisiológico de forma óptima tras la ejecución de la tarea. Teniendo en cuenta, a su vez, la óptima recuperación manifestada en los grupos de alto nivel de preparación y mayor expectativa, la activación fisiológica ha facilitado la adaptación de la persona para afrontar la tarea de forma más activa y con mayor implicación en la misma. Dicha activación parece indicar que las personas movilizan un conjunto de recursos fisiológicos, además de los cognitivos, para hacer frente a la situación de evaluación de sus capacidades. Tal como se informa en los trabajos de Gutiérrez y García (1999) y Palmero (1995), la activación fisiológica no se puede interpretar únicamente como la percepción de amenaza en una situación determinada, sino también como un requisito que permitirá a la persona mantener niveles de atención adecuados para recuperar la información almacenada previamente y poder alcanzar el objetivo deseado. Asimismo, nos parece pertinente indicar que la PS y PD son variables más decisivas que la FC a la hora de recoger diferencias de activación fisiológica ante la ejecución de una tarea al igual que se ha sugerido en algunos trabajos (Palmero, Breva y Landeta, 2002; Palmero, Gómez, Guerrero, Carpi, Díez y Diago, 2007). Y, por otra parte, entre la PS y PD, la primera parece ser un índice más representativo de la activación que la PD, hecho que puede ser constatado cuando apreciamos la variabilidad de los perfiles manifestados en ambos grupos de alta-baja expectativa y nivel de preparación.
En síntesis, cabe destacar que la realización de la tarea permite ajustar las expectativas de logro, ya que informa a la persona de cuál ha sido su ejecución y cuál es el resultado a alcanzar de forma más probable una vez realizada la tarea. Así, también la ejecución contribuye a valorar la preparación percibida de forma más ajustada.

La diferente activación fisiológica manifestada por los grupos con alta-baja expectativa de logro y nivel de preparación parece ser indicativa de la facilitación del afrontamiento de la tarea, del grado de motivación para conseguir determinados objetivos, más que atribuirla a niveles de ansiedad como puede suponerse en una situación de evaluación, como es un examen.

En cuanto a la variable de atribución, aunque se han podido detectar una débil relación con el resto de las variables, no podemos establecer ninguna afirmación que aporte conocimiento sobre su influencia en las expectativas de logro empleadas. La recogida de información sobre la atribución se ha realizado de forma muy general y con un único ítem que recogía información acerca de la atribución de logro situacional es decir, en qué medida los resultados esperados en el examen realizado se atribuía a la preparación realizada del mismo. Otros aspectos estables de la atribución como variable de personalidad, así como distintos factores situacionales relacionados con la dificultad de la tarea, entre otros, no han sido empleados lo que puede haber contribuido a los resultados estadísticos de este estudio. Además, el tipo de examen, la posibilidad de tener otra oportunidad para presentarse, la curiosidad acerca del experimento, 
entre otros factores, puede haber facilitado la participación en el mismo sin demasiada implicación o sentimiento de presión que la pérdida de convocatoria pueda suponer, lo cual puede haberse sumado a la nula relación significativa entre la variable de atribución y el resto de las variables empleadas.

En futuros trabajos será necesario abordar éstas y otras muchas cuestiones relacionadas con distintos factores emocionales para comprender otros aspectos que expliquen, de forma más amplia y detallada, el funcionamiento motivacional y el proceso seguido por los estudiantes ante la preparación de la materia a examinar, así como incorporar instrumentos de evaluación que permitan ajustar qué factores están influyendo en el desarrollo de las expectativas y de atribución y, si esta última, está relacionada con características fundamentalmente situacionales o de personalidad.

\section{Bibliografía}

Atkinson, J. W. (1964). An introduction to motivation. Princenton, NJ: D. Van Nostrand Co., Inc.

Beidel, D. C., Turner, S. M \& Dancu, C. V. (1985). Physiological, cognitive and behavioral aspects of social anxiety. Behavior Research and Therapy, 23, 109-117.

Blasco, T., Fernández-Castro, J., Doval, E., Moix, J., Rovira, T. \& Sanz, A. (1999). Competencia personal y estrés. Ansiedad y Estrés, 5, 261273.

Bruinsma, M. (2004). Motivation, cognitive processing and achievement in higher education. Learning and instruction. 14, 549-568.

De la Torre, C. \& Godoy, A. (2003). Diferencias individuales en las atribuciones causales de los alumnos y su influencia en el componente afectivo. Revista Mexicana de Psicología, 20, 237-246.
De la Peza Casares \& García, E. (2005). Relación entre variables cognitivo-emocionales y rendimiento académico: un estudio con universitarios. Recuperado de http://fsmorente.filos.ucm.es/publicacones/iberpsicologia/lisboa/peza/peza.htm.

Derakshan, N. \& Eysenck, M. W. (2001). Manipulation of focus of attention and its effects on anxiety in high-anxious individuals and repressors. Anxiety, Stress and Coping, 14, 173-191.

Do, S. L. \& Schallert, D. L. (2004). Emotions and classroom talk: Toward a model of the role of affect in students'experiences of classroom discussions. Journal of Educational Psychology, 96, 619-634.

Ekman, P. \& Shean, G. D. (1997). Habituation of cognitive and physiological arousal and social anxiety. Behaviour Research and Therapy, 29, 283-292.

Culler, R. \& Holahan, Ch. (1980). Test anxiety and academic performance: The effects of study-related behaviors. Journal of Educational Psychology, 72, 16-20.

Gómez, I. C., Carpi, A., Guerrero, C. \& Palmero, F. (2006). Ansiedad, miedo y defensividad: Efectos cardiovasculares en un contexto de evaluación académica. Revista de Psicología de la Salud, 18, 49-76.

González, D., Corral, V. \& Montaño, E. E. (2004). Percepción de las causas de logro académico en estudiantes de licenciatura. Revista Mexicana de Psicología, 21, 51-58.

Gore, P. A. (2006). Academic self-efficacy as a predictor of college outcomes: Two incremental validity studies. Journal of Career Assessment, 14, 92-115.

Guerrero, C. \& Palmero, F. (2006). Percepción de control, rendimiento y respuestas cardiovasculares. International Journal of Clinical and Health Psychology, 6, 145-168.

Gutierrez C. M. (1996). Ansiedad y deterioro cognitivo: Incidencia en el rendimiento académico. Ansiedad y Estrés, 2, 173-194. 
Gutierrez Calvo, M. \& García González, M.a D. (1999). Procesos cognitivos y ansiedad en situaciones de evaluación. Ansiedad y Estrés, 5, 229-245.

Heider, F. (1958). Thepsychology of interpersonal relations. Nueva York: John Wiley.

Manassero, M. A. \& Vázquez, A. (1995). Dimensionalidad de las causas percibidas en situaciones de éxito y fracaso escolar. Revista de Psicología Social, 2, 235-255.

Marshall, M. A. \& Brown, J. D. (2004). Expectations and realizations: The role of expectancies in achievement settings. Motivation \& Emotion, 28, 347-361.

Meijer, J. (2001). Stress in the relation between trait and state anxiety. Psychological Reports, 88, 947-964.

Palmero, F. (2005). Motivación: Conducta y Proceso. Revista Electrónica de Motivación y Emoción (REME), vol. VIII (20-21).

Palmero, F., Breva, A. \& Landeta, O. (2002). Hostilidad defensiva y reactividad cardiovascularen una situación de estrés real. Ansiedad y Estrés, 8, 115-142.

Palmero, F.; Gómez, C., Guerrero, C., Carpi, A., Díez, J. L. \& Diago, J. L. (2007). Hostilidad, psicofisología y salud cardiovascular. Avances en Psicología Latinoamericana/Bogotá (Colombia), 25, 22-43.

Rapee, R. M. (1993). The utilisation of working memory by worry. Research and Therapy, 31, 617-620.
Richard, E. M., Diefendorff, J. M. \& Martin, J. H. (2006) Revisiting the within-person selfefficacy and performance relation. Human Performance, 19, 67-87.

Rohrmann, S., Netter, P., Hennig, J. \& Hodapp, V. (2003). Repression-sensitization, gender, and discrepancies in psychobiological reactions to examination stress. Anxiety, Stress and Coping, 16, 321-329.

Shell, D. F., Murphy, C. C., \& Brunning, R. H. (1989). Self-efficacy and outcome expectancy mechanisms in reading and writing achievement. Journal of Education Psychology, 81, 91-100.

Shwarzwzer, C. \& Buchwald, P. (2003). Examination stress: measurement and coping. Anxiety, Stress and Coping, 16, 247-249.

Weiner, B. (1973). Theories of motivation. From mechanism to cognition. Chicago: Rand McNally.

Weiner, B. (1986). An attributional theory of motivation and emotion. Nueva York: Springer.

Weiner, B. (1980). A cognitive (attribution)emotion-action model of motivated behavior: An analysis of judgements of helpgiving. Journal of Personality and Social Psychology, 39, 186-200.

Zimmerman, B. J. (1999). Autoeficacia y desarrollo educativo. En A. Bandura (Ed.), Autoeficacia: como afrontamos los cambios de la sociedad actual (pp. 177-221). Bilbao: Desclee de Brouwer. 


\section{Apéndice}

Escala de medida de variables subjetivas

Nada

Mucha

1. Grado de preparación percibida para la ejecución del presente examen.

$\begin{array}{llllllllll}1 & 2 & 3 & 4 & 5 & 6 & 7 & 8 & 9 & 10\end{array}$

Suspenso Excelente

2. ¿Qué nota espera obtener en el presente examen?

$\begin{array}{lllllllllll}0 & 1 & 2 & 3 & 4 & 5 & 6 & 7 & 8 & 9 & 10\end{array}$

Suspenso

Excelente

3. Tras el examen realizado, ¿cuál es la nota que espera obtener?

$\begin{array}{lllllllllll}0 & 1 & 2 & 3 & 4 & 5 & 6 & 7 & 8 & 9 & 10\end{array}$

Muy en

desacuerdo

4. En qué medida cree que la nota del examen realizado depende de la preparación llevada a cabo

$\begin{array}{llllllllll}1 & 2 & 3 & 4 & 5 & 6 & 7 & 8 & 9 & 10\end{array}$

